\title{
Discussion on Operation Risk Analysis and Emergency Management Construction of Urban Large-scale Horticultural Exposition
}

\author{
Xinwei YIN ${ }^{1 \text { st }}$, Tong WANG ${ }^{2 d^{*}}$, Lianghai LV $^{3 \text { rd }}$, Baoqian DAI ${ }^{4 \text { th }}$, Yu WANG ${ }^{5 \text { th }}$ \\ ${ }^{1}$ Beijing Municipal Institute of Labour Protection Beijing, People's Republic of China \\ ${ }^{2}$ Beijing Municipal Institute of Labour Protection Beijing, People's Republic of China \\ ${ }^{3}$ Beijing Municipal Institute of Labour Protection Beijing, People's Republic of China \\ ${ }^{4}$ Beijing Key Laboratory of City Toxic, Harmful, Flammable and Explosive Hazard Control Technology Beijing, People's Republic of \\ China \\ ${ }^{5}$ Beijing Key Laboratory of City Toxic, Harmful, Flammable and Explosive Hazard Control Technology Beijing, People's Republic of \\ China
}

\begin{abstract}
With the development of society, the urban large-scale horticultural exposition has increasingly become one of the manifestations of enriching people's lives, but the issue of safety management has always been an important research topic. Taking Beijing World Horticultural Exposition as an example, this paper systematically analyzes the operation risk of large-scale horticultural exposition, and expounds on the characteristics of possible risks of the world horticultural exposition from the perspective of the existing nature of risks, and puts forward the construction plan of perfecting the responsibility system of emergency management. It aims to provide a theoretical reference for the operation risk analysis and emergency management of large-scale horticultural fairs.
\end{abstract}

\section{Introduction}

The Word Horticultural Exposition is the highest level of professional international exposition. Since the first Word Horticultural Exposition was held in Rotterdam, the Netherlands in 1960, 21 sessions have been held in the world. In recent years, China has actively participated in the bidding of international large-scale expositions. The successful Expos include Kunming in 1999, Shenyang in 2006, Taipei in 2010, Xi'an in 2011, Qingdao in 2014, Tangshan in 2016, Beijing in 2019, and Yangzhou Expo in 2021. The Modern World Horticultural Exposition has surpassed the concept of an exhibition in the general sense. It has become a grand festival in the world. It uses horticultural joint exhibitions of various countries as a link to promote the exchange of culture, economy, and human emotions, and pay more and more attention to high-tech Display and dissemination.

At present, the risk assessment of large-scale activities in China is still in its preliminary stage, especially the risk specific to large-scale activities is lack of overall comprehensive management evaluation. Fang Qianhua 1, Li Chungang 2 and others analyzed the main risks of the Beijing Olympics and explained how to improve the level of domestic security management services from three aspects: government management, security forecast analysis, and international cooperation; Teng Hui 3 mainly analyzed the passenger flow risk of Shanghai World Expo by using system dynamics and econometric model and proposed control measures to avoid the response risks; Hua Jingfeng 4 advocated the management principle of "who sponsored the large-scale event, who is responsible", and carried out security work around prevention and safety first; Feng Hongxin et al. 5 constructed an emergency response model for large-scale activities from the aspects of early-stage handling, linkage mechanism, and aftermath handling of large-scale event emergencies. As a typical and special large-scale activity, the World Horticultural Exposition will effectively stimulate the development of the urban trade and tourism services and environmental landscape. It will bring significant economic benefits and far-reaching social influence on the city, and at the same time, it will also be a major test to the government functions of the host area.

\section{Ease of Use Risk Analysis of the Operation of the World Horticultural Exposition}

Risk refers to the uncertain relationship between the purpose of production and output, and usually also represents the combination of the possibility of occurrence of a particularly dangerous event and the severity of its consequences 6. Large-scale horticultural expositions and other large-scale activities are prone to produce many unsafe factors Due to the characteristics of asymmetric

*Corresponding author: wangtong_ms@sina.com 
social participation and non-procedural decision-making in the dissemination of public information, which endangers the safety of regional public operations. Therefore, in the development of the operation plan, we must consider the potential safety hazards in the process of activity performance. Risks are not easy to be perceived directly. It is necessary to conduct field research based on existing data, comprehensively collect historical safety risk data, and analyze the factors leading to possible accidents and how to effectively avoid these risks. According to their types, risks can be divided into inherent risks, external risks, and management risks.

\subsection{Inherent Risk Factors}

Inherent risk refers to the intrinsic risk objectively existing by hazard sources. Its magnitude depends on the energy or quantity of hazard sources 7. Hazard sources are considered as carriers of dangerous substances or dangerous states, including locations, processes, activities, etc. 8. As a typical hazard carrier, the Expo has inherent safety risks in the process of early construction and later maintenance and operation, the safety of building structures, the safety of infrastructure, and the process of personnel operation. During the construction phase, it is particularly necessary to prevent object strikes, vehicle injuries, mechanical injuries, falls from heights, and fires among the 20 types of injuries. In the daily operation stage, we should pay attention to the potential danger of special equipment such as elevator pressure vessels, standardize the operation of personnel, and strengthen the daily inspection management. Inherent risk factors can be reduced to a controllable range through scientific means such as risk identification, risk analysis, and risk assessment.

\subsection{External Risk Factors}

From the perspective of intrinsic safety, external risks usually refer to environmental risks, generally including a bad physical environment or working environment 9. Due to the long time and wide range of participants, the Horticultural Exposition is often prone to trigger the above two risk factors and lead to unsafe incidents. It is necessary to prevent the impact of extreme weather on the operation safety of the expo, such as the sudden rainstorm caused by high temperature in summer, which is often accompanied by hidden safety problems. Also, the expo faces a lot of dynamic risk factors in the process of operation, tourist, vehicle, logistics, activities participants (foreign exhibitors, exhibitors, domestic exhibitors, sponsors) and several commercial activities will be held to the expo will bring many uncertain factors.

\subsection{Manage Risk Factors}

In Heinrich's Accident Causes Theory, unsafe behavior of people and unsafe state of things are the direct causes of accidents 10 . These risk factors are not only induced by environmental factors, but management link exposed defects often also play an important role. To achieve safety goals, safety management carries out decision-making, planning, organization, command, coordination, and control of safety through the function of management, so as to effectively identify and analyze various unsafe factors in the production process and avoid accidents 11 . The institutional setting, rules and regulations, leadership level, staff's physical and psychological state, knowledge and skills, working attitude, interpersonal relationship, and other factors will directly or indirectly affect the safe operation of an organization.

\section{Analysis of Risk Characteristics of the Expo}

As a modern travel area integrating entertainment, catering and accommodation, sightseeing and shopping, and business exhibitions, the Expo Park has a dense flow of people, a complex environment, lack of scientific risk data reports, management measures, and methods, so the public safety risk management problems are prominent. Compared with other general large-scale activities, the requirements for safe operation are also stricter. Beijing Word Horticultural Exposition also has its unique risk characteristics, mainly manifested in the following four main aspects:

\subsection{Long Exhibition Period}

The Beijing Expo spans from April to October 2019, with a duration of 162 days, which is a long-term large-scale event. Too long a holding period leads to an increase in hidden risk factors, followed by an increase in the difficulty of safety management.

\subsection{There Are Many Risk Factors and Dynamic Changes}

In the expo park, there is a concentrated layout of tourism and entertainment, garden activities, cultural creativity, modern business, and other boutique projects. Risk incentives are bound to be numerous and complex, and simple risks, complex risks, uncertain risks, and fuzzy risks are intertwined. The risk factors of large passenger flow are mainly divided into two categories: one is the trampling risk caused by excessive passenger flow. The other is the risk of trampling accidents derived from other emergencies. Various risk sources such as catering for the expo, gas stations, inclement weather, and pedestrian intersections on flyover roads can all reflect the characteristics of the four major emergencies. The mutual coupling, superposition, and derivation of multiple types of risk factors pose new challenges to the safety of large passenger flows. Therefore, it is necessary to conduct dynamic management according to the change of risk. Each risk identification will be dynamically updated, with the elimination of old risk sources and the discovery of new risk sources. 


\subsection{Passenger Flow Is Greatly Affected by the Current Traffic Situation}

During the Beijing International Horticultural Exposition held in Yanqing, tourists in Beijing and other ports mainly arrive at the venue through two expressways and S2 lines. However, the traffic capacity of main roads in Yanqing is limited. Once the traffic jam or traffic accident occurs in the main road network, it is easy to cause tourists to stay in the park, thus bringing the pressure of passenger flow relief. it is easy to cause tourists to stay in the park, which will increase the pressure of passenger flow.

\subsection{The Service Carrying Capacity of the Yanqing District Is Limited}

The Yanqing District covers an area of 200,000 hectares but has a population of only 300,000 . The daily operation capacity of the urban area is limited, including the reception capacity of tourists' accommodation, catering, and transportation. The limited-service carrying capacity of Yanqing affects the normal distribution of large passenger flow in the park to a certain extent. The limitedservice carrying capacity of Yanqing has affected the normal flow of passengers in the park to a certain extent.

\subsection{The Amplification Effect of Risks and Consequences}

Many tourists from home and abroad will travel to the Beijing Expo, which is another important window for Beijing to show its culture to the world. Once an emergency occurs in this area, it will have a bad social impact on Beijing's image as an international city.

\subsection{New Emergency Management Unit}

The Beijing Expo has been set up temporarily a new organization and management agency, the Beijing World Horticultural Exposition Affairs Coordination Bureau, which is responsible for the organization, construction, and management of the operation of the expo. At the same time, the Yanqing District Government, the Expo Site, is responsible for the territorial management of the peripheral operations of the Expo Park. For a new emergency management unit such as the expo, it is necessary to establish an emergency response mechanism involving the Beijing Municipal Government, the Expo Bureau, and the Yanqing District.

\subsection{The Emergency Response System and Mechanism Are Immature}

As the World Horticultural Exposition Bureau is a newly established institution, the establishment of the emergency organization and management system has not been tested, and the emergency working mechanism among various departments is still in the running-in period, which is a great challenge for emergency management.

\subsection{There Are Many Management Entities}

There are different management bodies in the World Park, such as the World Park Bureau, World Park Company, Vanke Company; There are many countries involved, including international and domestic provinces and cities of the exhibition hall of the horticultural expo large-scale activities drone performance group robot performance group sponsored by JD.com, the expo catering investment work is involved in more units and management bodies. Major events at the expo, such as drone performances and robot performances, are sponsored by JD.com. The horticultural expo catering investment work involves more units and management's main body. The security, traffic, parking, water, electricity, and heat infrastructure around the park also involve different responsibilities subjects. Therefore, the Beijing Expo has a multistructured organizational relationship between China and foreign countries, provinces and cities, government, and enterprises. So, its emergency management has significant diversification characteristics, which makes risk early warning and control coordination more difficult. Multidepartment management requires a clear division of responsible subjects, and the information communication and coordination mechanism between departments is particularly important, otherwise, it is easy to form management loopholes.

\section{Emergency Management Recommendations}

The expo will attract a large number of tourists from home and abroad to gather, and its safety risk assessment and safety prevention of large passenger flow is a large-scale systematic project, which involves many links and many factors, including the inherent risks of venue, equipment, and facilities, external risks influenced by weather, transportation, logistics, tourist, activities, and other factors, as well as the management risks generated by the coordination between the relevant industry management departments of the government and the relevant units of the event organizer. How to ensure the safety during the operation, the author thinks:

\subsection{Implement Safety Supervision Responsibilities.}

According to the characteristics of complex risks and difficult emergency management of the expo, combined with the organization and operation system of the expo, in order to effectively clarify the responsibility of safety supervision, the author proposes the following five principles of safety management:

\subsubsection{Leading by government management.}

The holding of the expo is highly valued by the Beijing Municipal and Yanqing District governments. It is also a test of Yanqing's ability to host world-class large-scale events and a test of the functions of the Yanqing District government. To successfully host this event, the 
government must lead the safe construction and smooth operation of the expo.

\subsubsection{Whoever manages is responsible, who supervises who is responsible.}

There are multiple management units involved in the park, and their main responsibility for safe production must be implemented; According to the functions, blocks, places, and equipment of the park, the operating subject and the security responsibility unit shall be divided accordingly. The responsibility of safety supervision can be divided from the aspects of government functions such as medical service, voluntary service, security, and stability maintenance.

\subsubsection{Classified and hierarchical management, and responsibility to the post.}

The expo has a long exhibition cycle, and the risk factors are varied and dynamic, which makes the management more difficult. According to the characteristics of incident risk, classify safety responsibility subjects according to department functions, and the work of risk management and emergency safety responsibility shall be effectively carried out.

\subsubsection{Block management, horizontally to the side and vertical to the bottom.}

Functional departments should clarify their respective management responsibilities and management authority to ensure that there are no blind spots and omissions in management.

\subsubsection{Up-and-down linkage, left-and-right collaboration.}

Up-and-down linkage means that the staff, venues, districts, departments, and leadership should be interconnected. Left-and-right cooperation means that the risk management and emergency response of many emergencies may not be a matter of a single department, a single area, or a single venue. They need to establish a collaboration mechanism and manage together.

\subsection{Improve The Emergency Plan System.}

In the safety management of large-scale activities, the primary work is to analyze the risk factors in the park and the severity of their consequences. The author adopts ISO 31000 risk assessment method 12-14, and evaluates the potential emergencies of the Beijing Expo from the perspective of four major types of emergencies (natural disasters, public health disasters, social security incidents). After field investigation and drawing on the experience of experts, it is determined that there are 49 possible emergencies in four major categories during the operation of the expo park. Then, combining with the nature of these emergencies, the possibility of occurrence and the severity of consequences of emergencies were analyzed, and 9 high-level risks, 36 medium-level risks, and 4 low-level risks were confirmed. The risk matrix of various events in the Beijing Expo Park is shown in Fig1

\section{Schematic Diagram of Risk Events for the Beijing Expo}

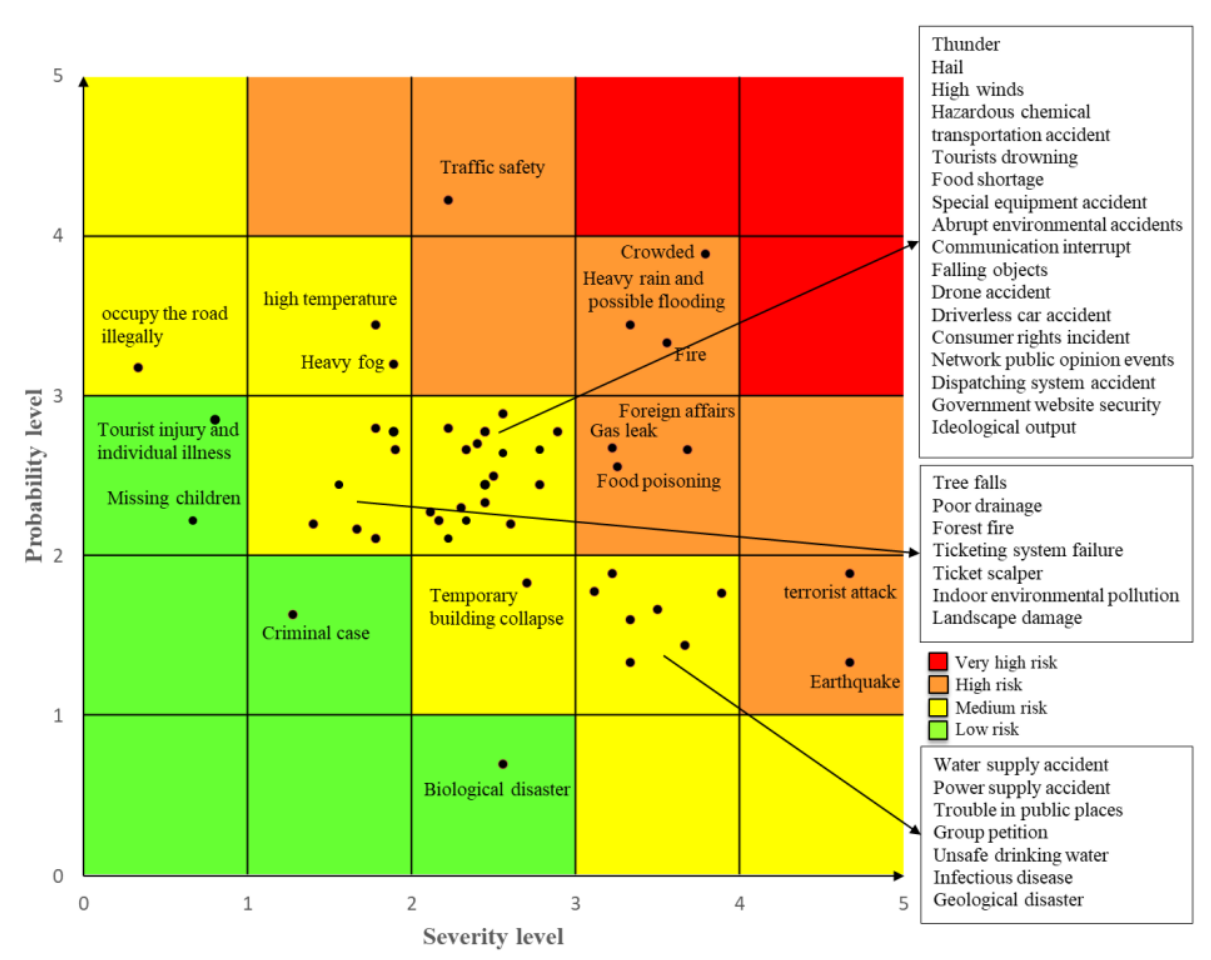

Fig1. Schematic diagram of various emergency risk matrix in the Beijing Expo park. 
Emergency plans are effective measures after the occurrence of uncertain events. Based on rehearsing the occurrence and evolution of accidents, dynamic analysis and timely response decision-making have high requirements for the ability to control the current situation. This requires the compiler to have overall awareness, logical deconstruction ability, and analyze the accident development trajectory to formulate emergency response measures at all levels when preparing corresponding contingency plans.

A complete plan system should include an overall emergency plan, a special plan for emergencies, and a site disposal program 15 . The overall emergency plan is a standardized procedure for organization and management, command and coordination of relevant emergency resources and emergency actions, which should include the organization system, monitoring and early warning, emergency response and rescue, aftercare, emergency support, and plan management; Special emergency plan is an emergency procedure compiled by various departments to deal with one or several types of emergency. It mainly contains four parts: emergency information reporting, advance disposal, emergency response, and follow-up disposal. For example, the Beijing Word Horticultural Exposition has a high risk of forest fires. Yanqing, a forested area, will face the test of summer during the expo. The special fire emergency plan is presented in Fig2

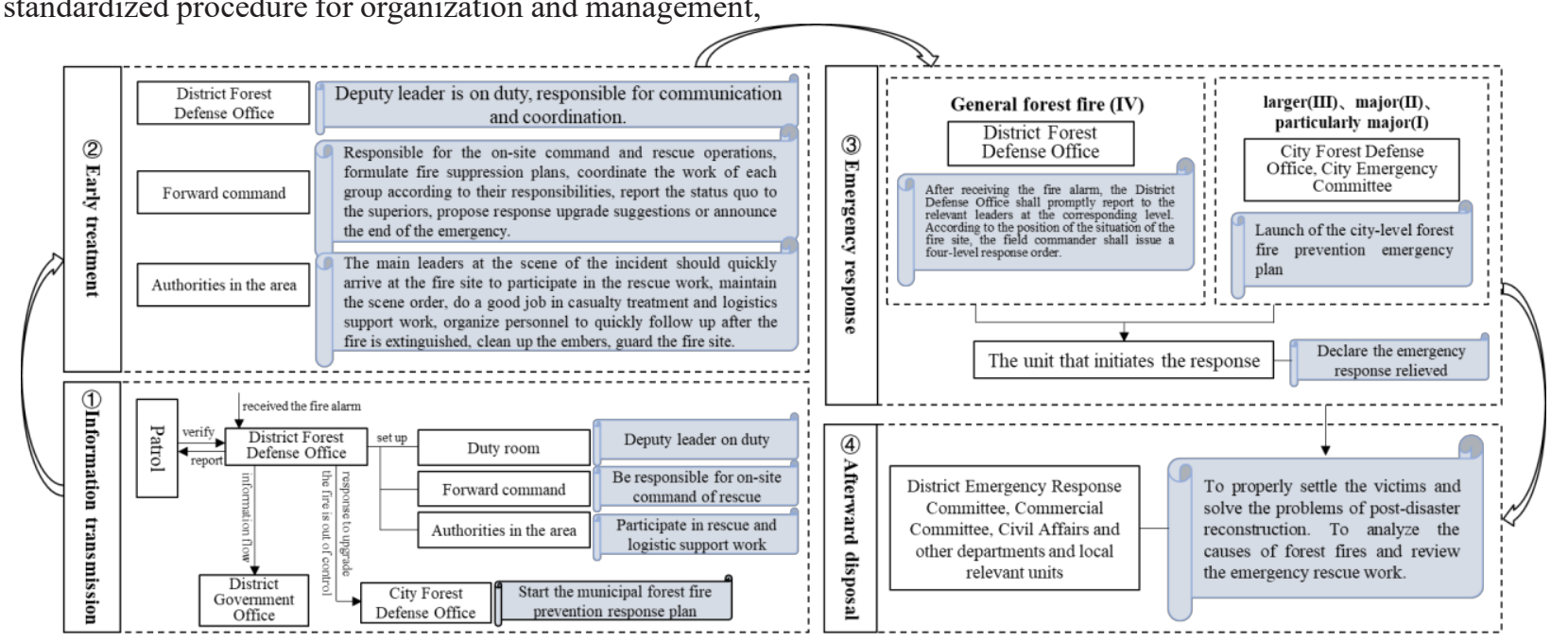

Fig2. Example: Forest fire special emergency plan disposal process.

The site disposal program is the work plan, guarantee plan, or operating procedure formulated by each department in response to the emergency based on the overall plan and special emergency plan. The content focuses on clarifying the specific responsible person, information submission, preliminary disposal, self-help, and mutual rescue, etc. The site disposal program is mainly prepared and implemented by the front management entities.

\subsection{Improve The Emergency Command Mechanism}

In the daily management activities, the normal emergency command mechanism should be established, and the wartime emergency needs should be met at the same time. A highly coordinated and unified emergency approach should be formed from the Ministry of State, Beijing Municipal Government, and Yanqing District Government to the expo park. The structure as shown in Fig3, will realize the rapid transition from the normal operating state to the wartime emergency state. Then based on the national emergency response standard, an emergency response classification system adapted to the characteristics of the expo park is established. For example, for the Beijing International Horticultural Expo, the author proposed a $3+3$ emergency management model, which divided the emergency level into three levels: ordinary, larger, major, and more, and then further divided the general emergency into three response levels. General Class I: The situation is relatively minor and no injuries were caused. The single department can handle emergencies independently; General level: the incident is relatively serious, which has caused or may cause multiple injuries (more than 3 people) or property losses, and requires joint treatment by multiple departments; General Level III: It belongs to the category of general incidents, but involves sensitive issues such as foreign-related issues, and needs to be reported to the higher level during on-site disposal. Larger emergencies: according to the circumstances, set up a temporary emergency command organization to coordinate the public security, armed police, traffic, fire protection, safety supervision, and other units to carry out emergency response work. Major and above emergencies: The Municipal Emergency Response Committee shall be responsible for directing and handling. According to the needs, the Municipal Party Secretary or the Mayor or the municipal leaders in charge shall rush to the scene and establish a temporary headquarters to direct and coordinate the relevant units and departments of the Municipal Special Headquarters for emergency handling. The organization within the Expo Bureau is responsible for handling general and larger incidents, while municipal agencies are responsible for handling major incidents and above. The "3+3" emergency response mode is shown in Fig4 


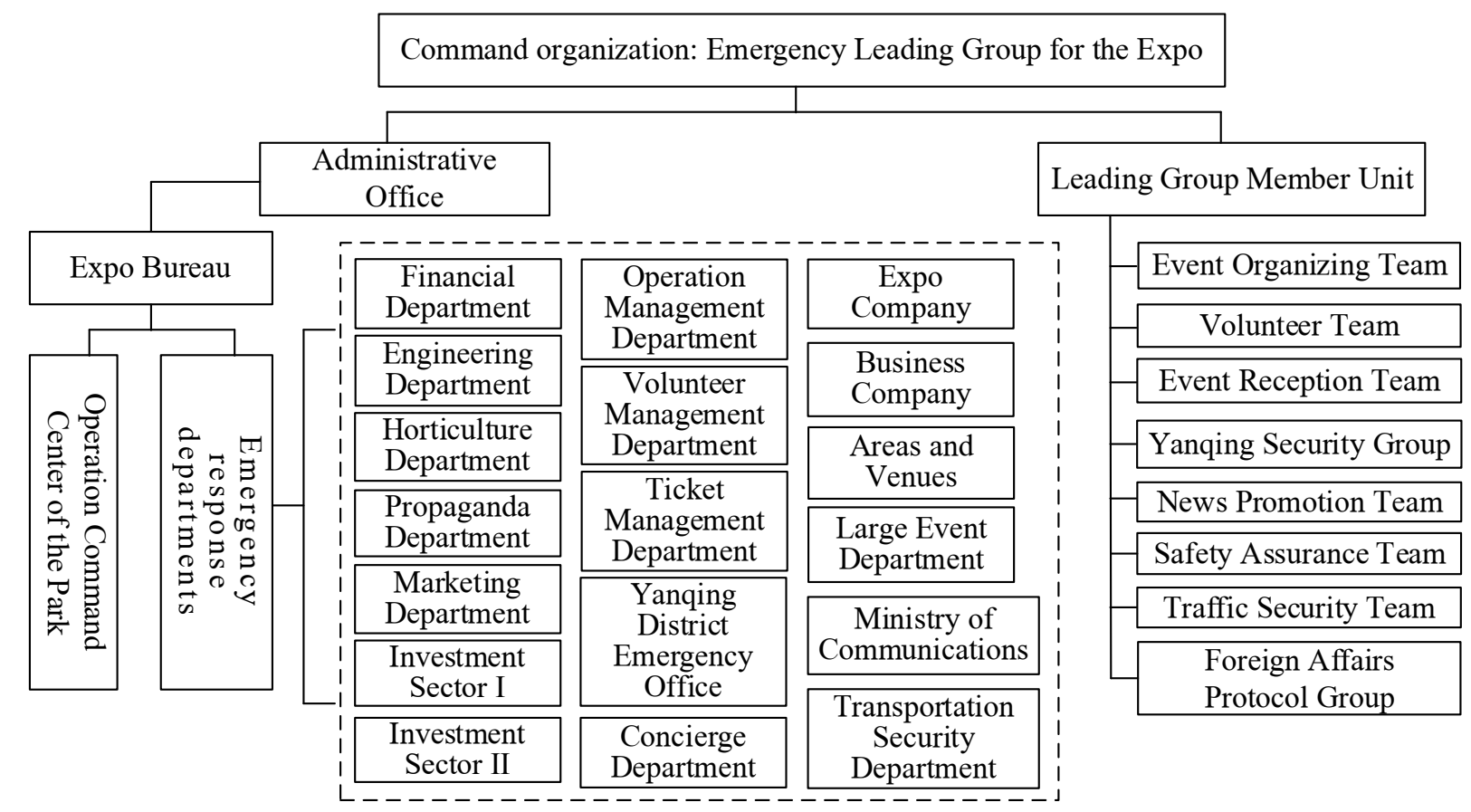

Fig3. Emergency organization structure of the Bejing Expo.

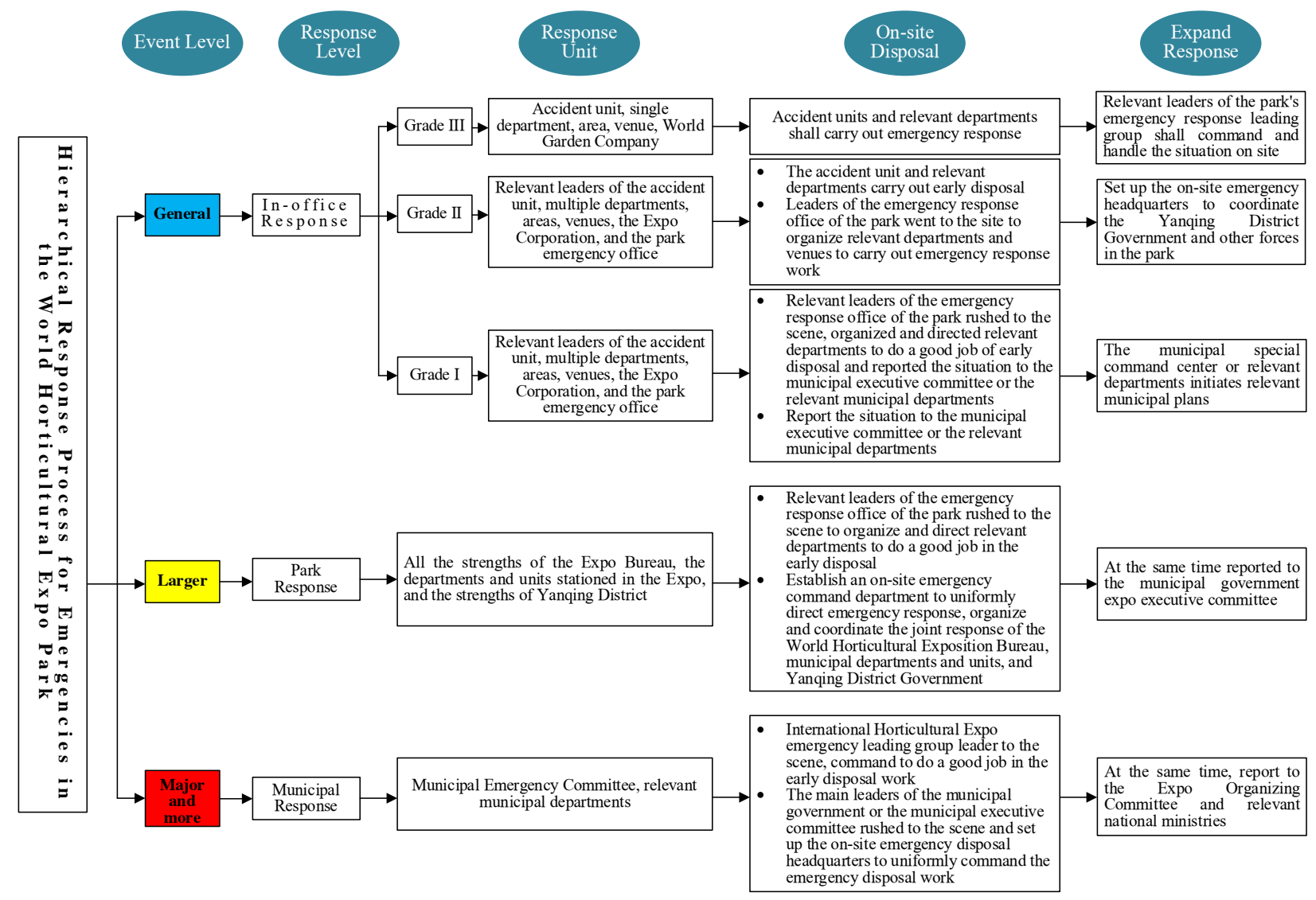

Fig4. Hierarchical response flow chart of emergencies in the Beijing Expo park.

\section{Acknowledgment}

The risk management of large-scale events such as the World Horticultural Exposition is a complex system engineering. This article takes the Beijing Expo as an example to condense safety management experience and elaborate on the possible existence of park operation management from the perspectives of inherent risks, external risks, and management risks. The author summarizes 8 aspects of operational risk and analyzes their risk characteristics.

Emergency management is an important aspect of risk management. This article describes the emergency 
management responsibility system from three aspects: implementation of supervisory responsibilities, formulation of emergency plans, and construction of emergency mechanisms. Based on the national emergency grading response standard, an emergency response grading system adapted to the characteristics of the expo was established in detail, and the $3+3$ emergency response model of the expo was proposed, which is intended to provide experience and reference for the managers of large-scale events such as large-scale exposition.

Supported by National Key R\&D Program of China (2018YFF0301003) and Enlightenment projects of BMILP.

\section{References}

1. FANG Qianhua. Research on service of security management for the Beijing Olympic Games. Journal of Wuhan Institute of Physical Education, 2007,41(1):29 32 .

2. LI Chungang. Analysis of the potential dangers of the safety of the Beijing 2008 Olympic Games. Beijing: Beijing Sport University.

3. TENG Hui. Operational risks and avoidance ideas in the process of hosting the 2010 Shanghai World Expo. Shanghai: Fudan University,2004.

4. HUA Jingfeng. Principles for the safety of large-scale events. Policing Studies,2004(11):33 35 .

5. FENG Hongxin, DU Mingxing. Construction of emergency disposal mode for large-scale security work. Journal of Jiangsu Police Institute, 2006,21(4):13 16 .

6. YIN Shuyue. Conceptual analysis of security and risk. Quality and Reliability,2011(3):50 52.

7. XU Ming, WU Zongzhi, LUO Yun. Axioms of safety technology for the work safety field. China Safety Science Journal,2015,21(1):3 8 .

8. FU Gui, LI Ya. Research on definition, content and classification of hazards in seven standards. China Safety Science Journal,2017,27(6):157 162 .

9. GUO Hanjun. Theory of accident causation based on internal-external factors and approaches for work safety. China Safety Science Journal,2007,17(7):46 53.

10. LIU Guoyu, LEI Ling. Study on the system of an accident-causing model and its general modeling methods and development trend[J]. Safety and Environmental Engineering,2013,20(1):138 142.

11. Tian Shuicheng, Jing Guoxun. Safety Management (2nd ed). Beijing: Machinery Industry Press, 2017:58 61.

12. ISO/FDIS 31000, Risk management-principles and guidelines.

13. ZHOU Rongyi, ZHONG An, REN Jingzhou. Comparative study on determining methods for safety integrity level of the safety system. Journal of Safety Science and Technology,2014,10(3):67 73.

14. LI Shuqing, YAN Zhi, DUAN Yu. Application of risk matrix in the classification of dangerous and hazardous factors. China Safety Science Journal,2010,20(4):83 87.

15. WANG Manli. Discussion on the main contents of the preparation of emergency rescue plan. Construction Safety,2019(4):45 48 . 